\title{
General Formula for Limit of Square Function at Infinity
}

\author{
Bonno Andri Wibowo ${ }^{1 *}$, Ikhsan Maulidi ${ }^{2}$,Windiani Erliana ${ }^{3}$ \\ 1,3 Institut Pertanian Bogor. Jalan Raya Dramaga, Bogor 16680, Indonesia. \\ 2 Universitas Syiah Kuala. Jalan Teuku Nyak Arief Darussalam, Banda Aceh 23111, Indonesia. \\ * Corresponding Author. E-mail: bonno1818@gmail.com
}

Received : 13-08-2018; Revised : 19-09-2018; Accepted : 30-09-2018

\begin{abstract}
Abstrak
Penentuan nilai limit dari suatu fungsi merupakan hal yang penting dilakukan. Sejatinya, nilai limit fungsi digunakan untuk melihat sifat fungsi di sekitar titik limitnya. Dalam artikel ini, diberikan rumus umum limit fungsi akar di takhinggaan. Rumus umum ini merupakan pengembangan dari rumus dasar dari limit fungsi akar di ketakhinggaan. Pengembangan yang dilakukan menggunakan teorema aljabar sederhana. Rumus umum limit fungsi akar di takhinggaan yang diperoleh mirip dengan rumus dasarnya.
\end{abstract}

Kata kunci: Fungsi Akar, Limit di Ketakhinggaan, Rumus Umum.

\begin{abstract}
Determination of the limit value of a function is an important things. Basically, the limit is used to look at the "properties" function value around the point. In this paper, we provide the general formula for the limit of square root function at infinite. This general formula comes from the development of a commonly known base formula. We use some simple algebra theorems to develop it. The result is very similar to the basic formula for limit of square root function at infinite.
\end{abstract}

Keywords: General Formula, Limit at Infinity, Square Function.

\section{INTRODUCTION}

Determination of the limit value of a function is an important aspect not only for calculus but also for other applied mathematics. Basically, the limit is used to look at the "properties" function value around the point. Limit at infinite is often used to see the convergence of a function. A function is said to be convergent if $\lim _{x \rightarrow \infty} f(x)=L<\infty$. One of research article about limit at infinity is about behaviour analysis for the solution of a problem (Kaewkhao, A. \& Intep, 2013). One particular form of square root function which is often discussed on popular calculus books is

$\lim _{x \rightarrow \infty} \sqrt{a x^{2}+b x+c}-\sqrt{p x^{2}+q x+r}$ $=\left\{\begin{array}{cl}\frac{b-q}{2 \sqrt{a}} & ; a=p \\ \infty & ; a>p \\ -\infty & ; a<p\end{array}\right.$

(Steward, 2011).

The Proof is omitted.

This study is a development of (1) to obtain the general formula of the square root function limit at infinite. Proof of general formula of the function by using algebraic properties of series and limit. Development is does gradually to see the pattern of proof.

Algebraic properties of series and limit which be used are

$$
\begin{gathered}
\sum_{i=1}^{n}\left(a_{i}+b_{i}\right)=\sum_{i=1}^{n} a_{i}+\sum_{i=1}^{n} b_{i} \\
\sum_{i=1}^{n} a_{i} p=p \sum_{i=1}^{n} a_{i} \\
\lim _{n \rightarrow \infty}\left(a_{n}+b_{n}\right)=\lim _{n \rightarrow \infty} a_{n}+\lim _{n \rightarrow \infty} b_{n}
\end{gathered}
$$

Proof. (Bartle, R.G. \& Shebert, 2010) 
Desimal, 1 (3), 2018 - 382

Bonno Andri Wibowo, Ikhsan Maulidi, Windiani Erliana

\section{METHODS}

This research is a literature review and the development of basic concepts of limit functions. Firstly, we provide some technical Lemmas which are useful for proving the main Theorem. Then the main theorem was given and we give some examples for result of this article.

\section{Technical Lemmas}

In this section, we provide some lemmas to be used in prooving of the Theorem

\section{Lemma 1:}

Let $a, b, c, p_{j}, q_{j}, r_{j} \in R$ and $a, p_{j}>0$.

If $\sqrt{a}=\sum_{j=1}^{n} \sqrt{p_{j}}$ then

$$
\begin{aligned}
& \lim _{x \rightarrow \infty}\left(\sqrt{a x^{2}+b x+c}-\sum_{j=1}^{n} \sqrt{p_{j} x^{2}+q_{j} x+r_{j}}\right) \\
& =\frac{b}{2 \sqrt{a}}-\sum_{j=1}^{n} \frac{q_{j}}{2 \sqrt{p_{j}}} .
\end{aligned}
$$

Proof:

$$
\begin{aligned}
& \lim _{x \rightarrow \infty}\left(\sqrt{a x^{2}+b x+c}-\sum_{j=1}^{n} \sqrt{p_{j} x^{2}+q_{j} x+r_{j}}\right) \\
& =\lim _{x \rightarrow \infty}\left(\sqrt{a} \sqrt{x^{2}+\frac{b x}{a}+\frac{c}{a}}-\sum_{j=1}^{n} \sqrt{p_{j} x^{2}+q_{j} x+r_{j}}\right) \\
& =\lim _{x \rightarrow \infty}\left(\sum_{j=1}^{n} \sqrt{p_{j}} \sqrt{x^{2}+\frac{b x}{a}+\frac{c}{a}}-\right. \\
& \left.\sum_{j=1}^{n} \sqrt{p_{j} x^{2}+q_{j} x+r_{j}}\right) \\
& =\lim _{x \rightarrow \infty}\left(\sum_{j=1}^{n} \sqrt{p_{j} x^{2}+\frac{p_{j} b x}{a}+\frac{p_{j} c}{a}}-\right. \\
& \left.\sum_{j=1}^{n} \sqrt{p_{j} x^{2}+q_{j} x+r_{j}}\right) \\
& =\lim _{x \rightarrow \infty} \sum_{j=1}^{n}\left(\sqrt{p_{j} x^{2}+\frac{p_{j} b x}{a}+\frac{p_{j} c}{a}}-\right. \\
& \left.\sqrt{p_{j} x^{2}+q_{j} x+r_{j}}\right) \\
& =\sum_{j=1}^{n} \lim _{x \rightarrow \infty}\left(\sqrt{p_{j} x^{2}+\frac{p_{j} b x}{a}+\frac{p_{j} c}{a}}-\right. \\
& \left.\sqrt{p_{j} x^{2}+q_{j} x+r_{j}}\right) \\
& =\sum_{j=1}^{n}\left(\frac{p_{j} b}{2 a \sqrt{p_{j}}}-\frac{q_{j}}{2 \sqrt{p_{j}}}\right) \\
& =\sum_{j=1}^{n} \frac{p_{j} b}{2 a \sqrt{p_{j}}}-\sum_{j=1}^{n} \frac{q_{j}}{2 \sqrt{p_{j}}} \\
& =\frac{b}{2 a} \sum_{j=1}^{n} \sqrt{p_{j}}-\sum_{j=1}^{n} \frac{q_{j}}{2 \sqrt{p_{j}}} \\
& =\frac{b}{2 a} \sqrt{a}-\sum_{j=1}^{n} \frac{q_{j}}{2 \sqrt{p_{j}}} \\
& =\frac{b}{2 \sqrt{a}}-\sum_{j=1}^{n} \frac{q_{j}}{2 \sqrt{p_{j}}} \text {. }
\end{aligned}
$$

This completes the proof of Lemma 1 .

\section{Lemma 2.}

Let $a_{i}, b, c, p_{j}, q_{j}, r_{j} \in R$ and $a_{i}, p_{j}>0$. If $\sum_{i=1}^{m} \sqrt{a_{i}}=\sum_{j=1}^{n} \sqrt{p_{j}}$ then

$$
\begin{aligned}
& \lim _{\substack{x \rightarrow \infty \\
\sum_{j=1}}}\left(\sum_{i=1}^{m} \sqrt{a_{i} x^{2}+a_{i} b x+a_{i} c}-\right. \\
& =\sum_{i=1}^{m} \frac{b_{i}}{2 \sqrt{a_{i}}}-\sum_{j=1}^{n} \frac{q_{j}}{2 \sqrt{p_{j}}}
\end{aligned}
$$

Proof:

$$
\begin{aligned}
& \lim _{x \rightarrow \infty}\left(\sum_{i=1}^{m} \sqrt{a_{i} x^{2}+a_{i} b x+a_{i} c}-\right. \\
& \left.\sum_{j=1}^{n} \sqrt{p_{j} x^{2}+q_{j} x+r_{j}}\right) \\
& =\lim _{x \rightarrow \infty}\left(\sum_{i=1}^{m} \sqrt{a_{i}} \sqrt{x^{2}+b x+c}-\right. \\
& \left.\sum_{j=1}^{n} \sqrt{p_{j} x^{2}+q_{j} x+r_{j}}\right) \\
& =\lim _{x \rightarrow \infty}\left(\sum_{j=1}^{n} \sqrt{p_{j}} \sqrt{x^{2}+b x+c}-\right. \\
& \left.\sum_{j=1}^{n} \sqrt{p_{j} x^{2}+q_{j} x+r_{j}}\right) \\
& =\lim _{x \rightarrow \infty}\left(\sum_{j=1}^{n} \sqrt{p_{j} x^{2}+b p_{j} x+c p_{j}}-\right. \\
& \left.\sum_{j=1}^{n} \sqrt{p_{j} x^{2}+q_{j} x+r_{j}}\right) \\
& =\lim _{x \rightarrow \infty} \sum_{j=1}^{n}\left(\sqrt{p_{j} x^{2}+b p_{j} x+c p_{j}}-\right. \\
& \left.\sqrt{p_{j} x^{2}+q_{j} x+r_{j}}\right) \\
& =\sum_{j=1}^{n} \lim _{x \rightarrow \infty}\left(\sqrt{p_{j} x^{2}+b p_{j} x+c p_{j}}-\right. \\
& \left.\sqrt{p_{j} x^{2}+q_{j} x+r_{j}}\right) \\
& =\sum_{j=1}^{n}\left(\frac{b p_{j}}{2 \sqrt{p_{j}}}-\frac{q_{j}}{2 \sqrt{p_{j}}}\right) \\
& =\sum_{j=1}^{n} \frac{b p_{j}}{2 \sqrt{p_{j}}}-\sum_{j=1}^{n} \frac{q_{j}}{2 \sqrt{p_{j}}} \\
& =\frac{b}{2} \sum_{j=1}^{n} \sqrt{p_{j}}-\sum_{j=1}^{n} \frac{q_{j}}{2 \sqrt{p_{j}}} \\
& =\frac{b}{2} \sum_{i=1}^{m} \sqrt{a_{i}}-\sum_{j=1}^{n} \frac{q_{j}}{2 \sqrt{p_{j}}} \\
& =\sum_{i=1}^{m} \frac{b \sqrt{a_{i}}}{2}-\sum_{j=1}^{n} \frac{q_{j}}{2 \sqrt{p_{j}}} .
\end{aligned}
$$

This completes the proof of Lemma 2 .

\section{RESULTS AND DISCUSSION \\ Theorem}

Let $a_{i}, b_{i}, c_{i}, p_{j}, q_{j}, r_{j}, b, c \in R$ and $a_{i}, p_{i}>0$ also

let $b_{i}=b a_{i}$ and $c_{i}=c a_{i}$.

Then

$$
\begin{aligned}
& \lim _{x \rightarrow \infty}\left(\sum_{i=1}^{m} \sqrt{a_{i} x^{2}+b_{i} x+c_{i}}-\right. \\
& \left.\sum_{j=1}^{n} \sqrt{p_{j} x^{2}+q_{j} x+r_{j}}\right) \\
& =\left\{\begin{array}{cc}
\sum_{i=1}^{m} \frac{b_{i}}{2 \sqrt{a_{i}}}-\sum_{j=1}^{n} \frac{q_{j}}{2 \sqrt{p_{j}}} & ; \sum_{i=1}^{m} \sqrt{a_{i}}=\sum_{j=1}^{n} \sqrt{p_{j}} \\
\infty & ; \sum_{i=1}^{m} \sqrt{a_{i}}>\sum_{j=1}^{n} \sqrt{p_{j}} \\
-\infty & ; \sum_{i=1}^{m} \sqrt{a_{i}}<\sum_{j=1}^{n} \sqrt{p_{j}}
\end{array}\right.
\end{aligned}
$$

Proof:

Case 1: $\sum_{i=1}^{m} \sqrt{a_{i}}=\sum_{j=1}^{n} \sqrt{p_{j}}$

$$
\begin{aligned}
& \lim _{x \rightarrow \infty}\left(\sum_{i=1}^{m} \sqrt{a_{i} x^{2}+b_{i} x+c_{i}}-\right. \\
& \left.\sum_{j=1}^{n} \sqrt{p_{j} x^{2}+q_{j} x+r_{j}}\right) \\
& \quad=\lim _{x \rightarrow \infty}\left(\sum_{i=1}^{m} \sqrt{a_{i} x^{2}+a_{i} b x+a_{i} c}-\right. \\
& \left.\sum_{j=1}^{n} \sqrt{p_{j} x^{2}+q_{j} x+r_{j}}\right)
\end{aligned}
$$

Substitute (2) to (5)

$$
=\sum_{i=1}^{m} \frac{b \sqrt{a_{i}}}{2}-\sum_{j=1}^{n} \frac{q_{j}}{2 \sqrt{p_{j}}}
$$

From (3) we have 
Desimal, 1 (3), 2018 - 383

Bonno Andri Wibowo, Ikhsan Maulidi, Windiani Erliana

$$
\begin{aligned}
& =\sum_{i=1}^{m} \frac{b_{i}}{a_{i}} \frac{\sqrt{a_{i}}}{2}-\sum_{j=1}^{n} \frac{q_{j}}{2 \sqrt{p_{j}}} \\
& =\sum_{i=1}^{m} \frac{b_{i}}{2 \sqrt{a_{i}}}-\sum_{j=1}^{n} \frac{q_{j}}{2 \sqrt{p_{j}}} .
\end{aligned}
$$

Case 2: $\sum_{i=1}^{m} \sqrt{a_{i}}>\sum_{j=1}^{n} \sqrt{p_{j}}$

Let: $\sum_{i=1}^{m} \sqrt{a_{i}}=\sum_{j=1}^{n} \sqrt{p_{j}}+K$ with $K>0$

$\lim _{x \rightarrow \infty}\left(\sum_{i=1}^{m} \sqrt{a_{i} x^{2}+b_{i} x+c_{i}}-\right.$

$\left.\sum_{j=1}^{n} \sqrt{p_{j} x^{2}+q_{j} x+r_{j}}\right)$

$=\lim _{x \rightarrow \infty}\left(\sum_{i=1}^{m} \sqrt{a_{i} x^{2}+a_{i} b x+a_{i} c}-\right.$

$\left.\sum_{j=1}^{n} \sqrt{p_{j} x^{2}+q_{j} x+r_{j}}\right)$

$=\lim _{x \rightarrow \infty}\left(\sum_{i=1}^{m} \sqrt{a_{i}} \sqrt{x^{2}+b x+c}-\right.$

$\left.\sum_{j=1}^{n} \sqrt{p_{j} x^{2}+q_{j} x+r_{j}}\right)$

$=\lim _{x \rightarrow \infty}\left(\left(\sum_{j=1}^{n} \sqrt{p_{j}}+K\right) \sqrt{x^{2}+b x+c}-\right.$

$\left.\sum_{j=1}^{n} \sqrt{p_{j} x^{2}+q_{j} x+r_{j}}\right)$

$=\lim _{x \rightarrow \infty}\left(\sum_{j=1}^{n} \sqrt{p_{j} x^{2}+b p_{j} x+c p_{j}}+\right.$

$\left.\sqrt{K x^{2}+b K x+c K}-\sum_{j=1}^{n} \sqrt{p_{j} x^{2}+q_{j} x+r_{j}}\right)$

$=\lim _{x \rightarrow \infty}\left[\sqrt{K x^{2}+b K x+c K}+\right.$

$\left.\sum_{j=1}^{n}\left(\sqrt{p_{j} x^{2}+b p_{j} x+c p_{j}}-\sqrt{p_{j} x^{2}+q_{j} x+r_{j}}\right)\right]$

$=\infty$.

Case 3: $\sum_{i=1}^{m} \sqrt{a_{i}}<\sum_{j=1}^{n} \sqrt{p_{j}}$

Let: $\sum_{i=1}^{m} \sqrt{a_{i}}=\sum_{j=1}^{n} \sqrt{p_{j}}-L$ with $L>0$

$\lim _{x \rightarrow \infty}\left(\sum_{i=1}^{m} \sqrt{a_{i} x^{2}+b_{i} x+c_{i}}-\right.$

$\left.\sum_{j=1}^{n} \sqrt{p_{j} x^{2}+q_{j} x+r_{j}}\right)$

$=\lim _{x \rightarrow \infty}\left(\sum_{i=1}^{m} \sqrt{a_{i} x^{2}+a_{i} b x+a_{i} c}-\right.$

$\left.\sum_{j=1}^{n} \sqrt{p_{j} x^{2}+q_{j} x+r_{j}}\right)$

$=\lim _{x \rightarrow \infty}\left(\sum_{i=1}^{m} \sqrt{a_{i}} \sqrt{x^{2}+b x+c}-\right.$

$\left.\sum_{j=1}^{n} \sqrt{p_{j} x^{2}+q_{j} x+r_{j}}\right)$

$=\lim _{x \rightarrow \infty}\left(\left(\sum_{j=1}^{n} \sqrt{p_{j}}-L\right) \sqrt{x^{2}+b x+c}-\right.$

$\left.\sum_{j=1}^{n} \sqrt{p_{j} x^{2}+q_{j} x+r_{j}}\right)$

$=\lim _{x \rightarrow \infty}\left(\sum_{j=1}^{n} \sqrt{p_{j} x^{2}+b p_{j} x+c p_{j}}-\right.$

$\left.\sqrt{L x^{2}+b L x+c L}-\sum_{j=1}^{n} \sqrt{p_{j} x^{2}+q_{j} x+r_{j}}\right)$

$=\lim _{x \rightarrow \infty}\left[-\sqrt{L x^{2}+b L x+c L}+\right.$

$\left.\sum_{j=1}^{n}\left(\sqrt{p_{j} x^{2}+b p_{j} x+c p_{j}}-\sqrt{p_{j} x^{2}+q_{j} x+r_{j}}\right)\right]$

$=-\infty$.

This completes the proof of Theorem.

\section{Appendix}

In this section, the authors provide examples of the use of the obtained formula and compare the results with Laurent series approach.

\section{Example of Lemma 1}

$\operatorname{Lim}_{x \rightarrow \infty}\left(\sqrt{4 x^{2}+x-1}-\sqrt{x^{2}-x+1}-\sqrt{x^{2}-2 x+3}\right)$ $=\frac{1}{2 \sqrt{4}}-\frac{-1}{2 \sqrt{1}}-\frac{-2}{2 \sqrt{1}}=\frac{7}{4}$.

Laurent series approach:

$$
\begin{aligned}
& \sqrt{4 x^{2}+x-1}-\sqrt{x^{2}-x+1}-\sqrt{x^{2}-2 x+3} \\
& =\frac{7}{4}-\frac{105}{64 x}-\frac{591}{512 x^{2}}+O\left(\left(\frac{1}{x}\right)^{3}\right) . \\
& \quad \text { So } \\
& \operatorname{Lim}_{x \rightarrow \infty}\left[\sqrt{4 x^{2}+x-1}-\sqrt{x^{2}-x+1}-\sqrt{x^{2}-2 x+3}\right] \\
& =\operatorname{Lim}_{x \rightarrow \infty} \frac{7}{4}-\frac{105}{64 x}-\frac{591}{512 x^{2}}+O\left(\left(\frac{1}{x}\right)^{3}\right)=\frac{7}{4} .
\end{aligned}
$$

\section{Example of Lemma 2/Theorem}

$\operatorname{Lim}_{x \rightarrow \infty}\left[\begin{array}{c}\left(\sqrt{x^{2}+2 x-1}+\sqrt{64 x^{2}+128 x-64}\right)- \\ \left(\sqrt{4 x^{2}-3 x+3}+\sqrt{9 x^{2}+5 x+10}+\sqrt{16 x^{2}+8 x-3}\right)\end{array}\right]$

$=\frac{2}{2 \sqrt{1}}+\frac{128}{2 \sqrt{64}}-\frac{-3}{2 \sqrt{4}}-\frac{5}{2 \sqrt{9}}-\frac{8}{2 \sqrt{16}}=\frac{95}{12}$.

Laurent series approach:

$\left(\sqrt{x^{2}+2 x-1}+\sqrt{64 x^{2}+128 x-64}\right)-$

$\left(\sqrt{4 x^{2}-3 x+3}+\sqrt{9 x^{2}+5 x+10}+\right.$

$\left.\sqrt{16 x^{2}+8 x-3}\right)$

$=\frac{95}{12}-\frac{18421}{1728 x}+\frac{1129361}{124416 x^{2}}+O\left(\left(\frac{1}{x}\right)^{3}\right)$.

So

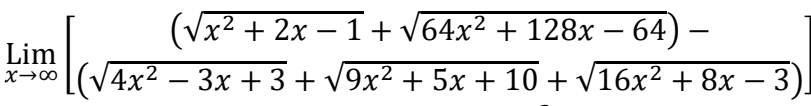

$=\operatorname{Lim}_{x \rightarrow \infty} \frac{95}{12}-\frac{18421}{1728 x}+\frac{1129361}{124416 x^{2}}+O\left(\left(\frac{1}{x}\right)^{3}\right)=\frac{95}{12}$.

Other Example (When $b_{i}$ and $c_{i}$ values which do not depend on $a_{i}$ values)

$\operatorname{Lim}_{x \rightarrow \infty}\left[\begin{array}{c}\left(\sqrt{x^{2}+2 x-1}+\sqrt{64 x^{2}+16 x-64}\right)- \\ \left(\sqrt{4 x^{2}-3 x+3}+\sqrt{9 x^{2}+5 x+10}+\sqrt{16 x^{2}+8 x-3}\right)\end{array}\right]$

$=\frac{2}{2 \sqrt{1}}+\frac{16}{2 \sqrt{64}}-\frac{-3}{2 \sqrt{4}}-\frac{5}{2 \sqrt{9}}-\frac{8}{2 \sqrt{16}}=\frac{11}{12}$.

Laurent series approach:

$\left(\sqrt{x^{2}+2 x-1}+\sqrt{64 x^{2}+128 x-64}\right)-$

$\left(\sqrt{4 x^{2}-3 x+3}+\sqrt{9 x^{2}+5 x+10}+\right.$

$\left.\sqrt{16 x^{2}+8 x-3}\right)=\frac{11}{12}-\frac{11617}{1728 x}+\frac{197213}{124416 x^{2}}+O\left(\left(\frac{1}{x}\right)^{3}\right)$.

So

$\operatorname{Lim}_{x \rightarrow \infty}\left[\frac{\left(\sqrt{x^{2}+2 x-1}+\sqrt{64 x^{2}+128 x-64}\right)-}{\left(\sqrt{4 x^{2}-3 x+3}+\sqrt{9 x^{2}+5 x+10}+\sqrt{16 x^{2}+8 x-3}\right)}\right]$

$=\operatorname{Lim}_{x \rightarrow \infty} \frac{11}{12}-\frac{11617}{1728 x}+\frac{197213}{124416 x^{2}}+O\left(\left(\frac{1}{x}\right)^{3}\right)=\frac{11}{12}$.

\section{CONCLUSION AND SUGGESTION}

The general formula of the square root function limit at infinte in (4) is very similar with the basic formula of square root function limit in (1). The author strongly 
believes that although $b_{i}$, and $c_{i}$ are independent of $a_{i}$ values, we still can use this general formula. In the appendix section, the author gives examples of limit function and compare the results of calculations with the calculation of the limit value using Laurent series approach method (Rodriguez, R.E, Kra, I.,\& Gilman, 2012).

Further researchs that can be done is to provide a mathematical proof for $b_{i}$ and $c_{i}$ values which do not depend on $a_{i}$ values or giving a counter example for the general formula.

\section{REFERENCES}

Bartle, R.G. \& Shebert, D. R. (2010). Introduction to Real Analysis. (I. John Wiley \& Sons, Ed.) (4th ed.). Urbana.

Kaewkhao, A. \& Intep, S. (2013). End Behavior Analysis for Solutions of Limit at Infinity. International Journal of Pure and Applied Mathematics, 89(1), 89-94.

Rodriguez, R.E, Kra, I.,\& Gilman, I. . (2012). Complex Analysis: In the Spirit of Lipman Bers. (Springer, Ed.) (2nd ed.). New York.

Steward, J. (2011). Calculus. (C. Learning, Ed.) (7th ed.). Belmont. 